\title{
Kinoform synthesis as an improved method to form a concealed image in optical security devices
}

\author{
Eu. Braginets' ${ }^{1}$, V. Girnyk ${ }^{2}$, S. Kostyukevych ${ }^{3}$, V. Kurashov ${ }^{1}$, N. Moskalenko ${ }^{3}$, A. Soroka ${ }^{1}$ \\ ${ }^{I}$ Taras Shevchenko Kyiv National University (Ukraine) \\ ${ }^{2}$ Optronics (Ukraine) \\ ${ }^{3}$ V. Lashkaryov Institute of Semiconductor Physics, NAS of Ukraine (Ukraine)
}

\begin{abstract}
It is well known that one of the basic functions of security holograms is the maximal complication of their non-authorized reproduction, in other words counterfeiting. To solve the problem, concealed images that can be observed only under special conditions are placed into a structure of the hologram. A popular way to place concealed image in Diffractive Optically Variable Image Device (DOVID) is integration into DOVID's structure of a Concealed Laser-Readable Image (CLRI). Traditionally CLRI is a 2D Computer-Generated Hologram (2D CGH), which is a digitized Interference Fringe Data (IFD) structure, computed under the scheme of Fourierhologram synthesis. Such hologram provides inspection of the second level with portable laser reading devices. While it is being read, two (+/- 1 order of diffraction) identical images are formed. It is very interesting to achieve a $\mathrm{CGH}$, which restores the image only in one diffractive order or two different images in +1 and -1 orders of diffraction.
\end{abstract}

Keywords: concealed image, kinoform methods, 2D CGH, CLR.

Manuscript received 20.09.06; accepted for publication 23.10.06.

\section{Introduction}

Computer generated Fourier holograms (Fourier CGH) are widely used for the concealed image recording in holographic protective elements. Decoding of such holograms is realized in an optical way with use of coherent decoders. It allows to strengthen a resistance of protective elements to forgery and increase an objecttivity of their identification procedure.

Generally, a problem of synthesis and Fourier spectrum recording comes to calculation of $\mathbf{A}(\xi, \eta)$ function in the frequency plane by specified distribution of complex amplitudes a $(x, y)$ in the coordinate area and to record the received distribution of complex wave field on a real physical carrier.

$\mathbf{A}(\xi, \eta)=\int_{-\infty}^{\infty} \int_{\mathbf{a}} \mathbf{a}(x, y) \exp (2 \pi i \xi x) \exp (2 \pi i \eta y) d x d y$,

where a $(x, y)=a(x, y) \exp (i \varphi(x, y))$.

One of the main problems, which we face with when recording Fourier holograms, is a recording of complex value.

There are two in principle different ways to solve this problem, namely: use of various methods to encode the Fourier spectrum, which allows to proceed from the complex quantity of field distribution in a hologram registration plane to the real quantity [1-3]. The methods in which the phase is encoded by means of space carrier, is of the largest interest. They combine more naturally with modern recording devices. We call this the holographic methods optimized for digital synthesis. The second way is direct recording the Fourier spectrum on double-layer amplitude-phase registering media [4-5]. This method is technologically possible, but it is not acceptable for integration of such structures into the holographic protective elements. However, it is possible its cut-down version when in the computed Fourier spectrum the amplitude is forcibly assumed to be equal to a constant and phase recording is realized, for example, by phase modulation of a geometric relief of the recording medium.

This method was called as a kinoform by creators. Kinoform transmission can be recorded in the following form:

$A[r, s]=$ const $\cdot \exp \{i \varphi[r, s]\}$

where $0 \leq \varphi[r, s] \leq 2 \pi$ along all plane $[r, s]$. 
It is necessary to mention that digital Fourier holograms are flat and act as a flat 2D diffraction grating. Thus, they restore two diffraction orders - two conjugated images. It applies restriction on the recorded object - the function a $(x, y)$ must satisfy the causality condition and turn into zero at $x<0$ :

$$
\mathbf{a}(x, y)=a(x, y) \exp (i \varphi(x, y))=0 \text { at } x<0 .
$$

Otherwise, mutual overlay of the real and conjugated images will occur.

Studied in this work is the possibility to enlarge opportunities of synthesis and recording the digital Fourier spectra removing restrictions connected with the causality condition.

From the practical standpoint, it will allow to increase the resistance of the hologram to falsification (synthesis algorithm changes, demands to the technological process and materials are increased), to increase the intensity of the image restored (owing to energy re-distribution) and to enlarge the possibilities for design solutions (owing to removing the restrictions (3)).

Below we examine two approaches for solution of this problem: use of quadrature holograms and kinoform methods of encoding.

\section{Use of quadrature holograms for elimination of conjugated images}

When recording a traditional Fourier hologram on registering medium in accordance with a computed interference fringe data (IFD) an intensity distribution is recorded [7]

$$
I(x)=\left|1+\exp \left(-i \omega_{0} x\right)\right|^{2}=2\left(1+\cos \left(\omega_{0} x\right)\right)
$$

When restoring this interferogram with a plane wave falling normally, two images of the object appear a conjugated image and a real one. The physical reason of twin-image appearance is explained by the fact that such hologram has no information about the direction from which the object wave felt on the hologram (in contrast, for example, to Denisyuk thick-layer hologram where the direction of wave propagation is unambiguously registered in the medium).

From the mathematical point of view, appearance of two conjugated waves at the stage of hologram restoring (instead of one wave when recording) is explained by $\cos (\omega x)$ function parity that is integral part of the registered value. Fig. 1 shows the recording of the point-like light source (PLS) object hologram in the Leith scheme. $k$ is the wave vector of the object wave and $k_{0}$ is the wave vector of the reference wave. It is not possible to determine a growth direction of complex components $\exp \left(+i \omega_{0} x\right)$ and $\exp \left(-i \omega_{0} x\right)$, IFD for cases of the object wave falling at $\theta$ and $-\theta$ angles will be the same.

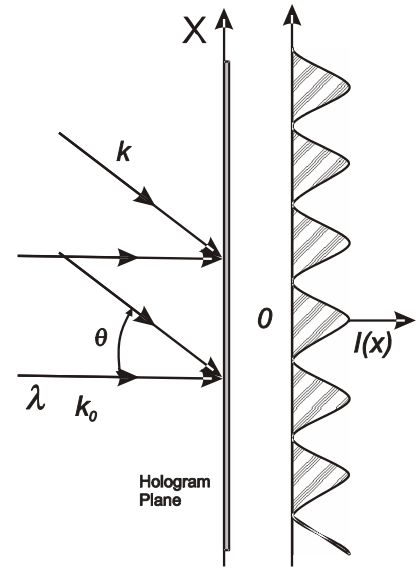

Fig. 1. Scheme of elementary Leith hologram recording. $k_{0}$ is the wave vector of the reference wave, $k$ is the wave vector of the object wave, $I(x)$ is the distribution of the light intensity in the hologram registration plane.

It is logically to suppose that, if to insert an optical element in this recording scheme, which would shift a phase of the reference wave by $\pi / 2$ radian, the same hologram of PLS contained already the sine (odd) function.

Such a hologram, where intensity distribution is described by $\sin \left(\omega_{0} x\right)$ function, is called a quadrature hologram. The diagram of recording of a primitive quadrature hologram according to the Leith scheme is shown in Fig. 2. Intensity distribution in this hologram is described by the following expression (5)

$$
I(x)=\left|\exp (i \pi / 2)+\exp \left( \pm i \omega_{0} x\right)\right|^{2}=2\left(1+\sin \left(\omega_{0 x}\right)\right) .
$$

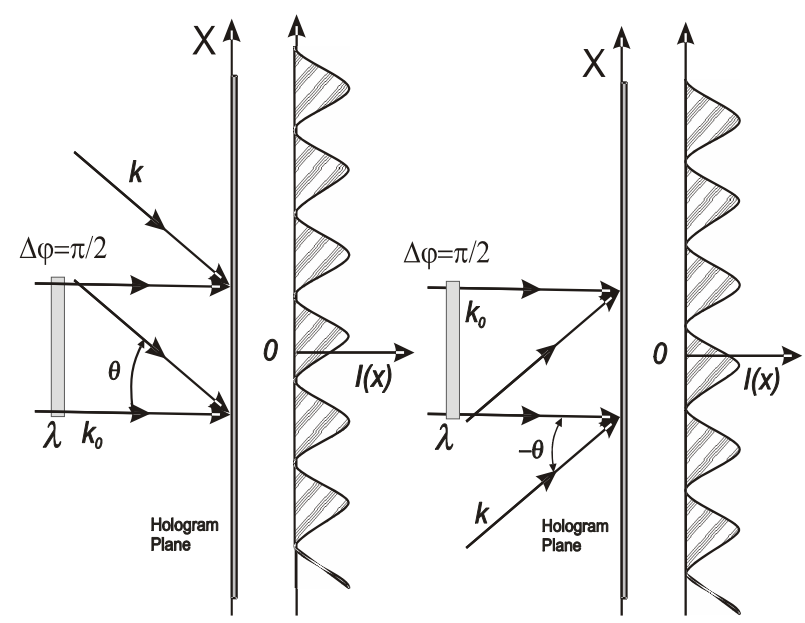

Fig. 2. Scheme of 2D quadrature hologram recording. A phase plate shifting wavefront phase on $\pi / 2$ is placed in the reference beam. In the upper picture the reference wave falls at $+\theta$ angle, in the lower one - at $-\theta$ angle. An appearance of the interference pattern depends on that, from which direction the reference wave comes. 
Eq. (5) shows that in any point with $x=0$, corresponding to the intensity maximum in the traditional hologram, the intensity in quadrature hologram will increase or fall depending on that, at which positive or negative angle the object wave falls on the hologram. To restore both holograms [8-10] (traditional or quadrature) are used, the wave phase restored by the quadrature hologram must be additionally shifted on $\lambda / 4$, thus, the total wave field will be as follows:

$u_{r}(x)=\left(1+\cos \omega_{0} x\right)+i\left(1 \pm \sin \omega_{0} x\right)=$

$=(1+i)+\exp \left( \pm \omega_{0} x\right)$.

As indicated in (6), when restoring, only one diffraction order remains. Combination of the traditional and quadrature holograms allows to register different intensity distribution in hologram plane depending on mutual source location of the object and reference waves.

Below you can see a computer algorithm flowchart for computation of such combined hologram (Fig. 3) and the results of the computer modelling (Fig. 4).

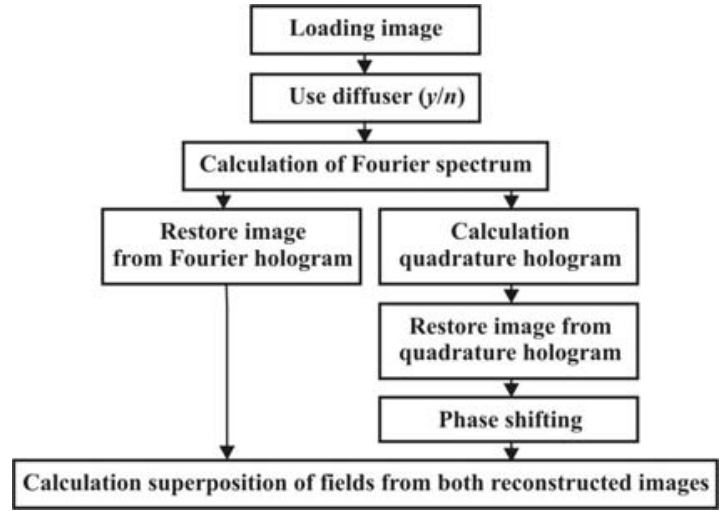

Fig. 3. Algorithm of computation and modelling of restoring of the combined hologram.

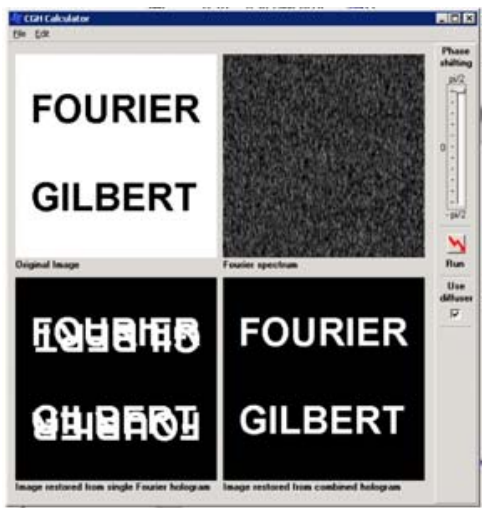

Fig. 4. Screen shot of the program on modelling restoration of the combined hologram. As object, words "FOURIER" and "GILBERT" in opposite semiplanes (left top figure) were used. The Fourier-interferogram with diffuser is shown in the top right image. Mathematically restored image shown in the left bottom figure (we can see two overplaced images), in the right bottom figure - image restored from the combined hologram (one order is restored only, overplacing is absent).

\section{Use kinoform methods for twin-image elimination}

As was mentioned above, the kinoform [6, 11, 12] represents a special type of a thin-layer hologram, where a complex amplitude of an object light wave $a(x, y)=$ $=A(x, y) \times \exp [i \varphi(x, y)]$ in the plane of hologram registration is almost constant on the module. In practice this situation takes place, when object has a diffused surface, or lighted with a diffused light or diffuser is installed on the way of object wave propagation. In such cases, the image of the object can be restored with use of the phase information $\varphi(x, y)$ only.

\subsection{Synthesis of the kinoform}

Synthesis of the kinoform was carried out according to the scheme of calculation of the Fourier-hologram. The object was defined in the object plane as a set of point light sources. Then, after the Fourier transformation, a mathematical analogue of the object wave in the hologram plane was calculated. As a basic assumption, a constancy of the module complex amplitude of the object wave $\mathbf{a}(x, y)=$ const $\cdot \exp [i \Delta \varphi(x, y)]$ was made, in addition for minimization losses related with rejection of the amplitude information in the algorithm of calculations a block of diffuser optimization was added. After calculation of the phase difference between object and reference waves in every point $(x, y)$ of the hologram plane, the result was normalized in such a manner that the phase function $(x, y)$ varies within $0-2 \pi$. As a result, we obtained 2D array consisting of phase discrete values

$$
\begin{aligned}
& \tilde{\varphi}(x, y)=\sum_{n=-N}^{N} \sum_{m=-M}^{M} \varphi_{n m}(\bmod 2 \pi) \delta(x-n \Delta x, y-m \Delta y), \\
& \varphi_{n m}(\bmod 2 \pi)=\varphi-2 \pi j \\
& j 2 \pi \leq \varphi \leq(j+1) 2 \pi, \quad j=0,1,2, \ldots
\end{aligned}
$$

The obtained array of phase values was used for kinoform recording on the phase recording medium.

The quality of the image restored from the kinoform mainly depends on two factors - value constancies of the module in the complex amplitude object wave and a performance of a condition of the phase coordination (2). The performance of a condition of the phase matching depends on accuracy of the transfer of calculated discrete values of the phase on the recording medium. The constancy of the amplitude value of the field in the plane of registration is provided owing to the insertion of the object diffuser. As a first approximation, the diffuser represents an array with random values of a phase (from 0 up to $2 \pi$ ).

This object-independent diffuser provides a rather constant object wave amplitude value in the field of the hologram registration, allows to obtain the value of SNR about 20 for the restored image. However, the granular structure of the image and a speckle around of the image 
remains. To eliminate these phenomena an algorithm of diffuser optimization was applied.

For the first time, this algorithm was described in $[13,14]$, where it is called an iterative algorithm of diffuser optimization. An essence of this algorithm is in the following: in the iterative cycle the initial array of the amplitude and phase values, which describes object is multiplied by the set of random values of the phase (diffuser).

The Fourier transform from the object (actually, mathematically restored image) is calculated. Then SNR at this step of iteration is calculated. The value of SNR is saved, then, after it, the next iteration step is made. If after the next iteration, SNR value is higher than the saved one, value of a phase diffuser in the given point is accepted corresponding to maximal SNR. The iterative cycle repeats itself or given number of times (or till achievement of the given SNR value). As a result SNR value was increased up to 30 .

With the purpose to get more constant values of the amplitude complex field in the hologram plane, we proposed to supplement this algorithm with an additional emphasis of the object amplitude [15-19].

\subsection{Mathematical modelling of image restoring processes}

A direct analysis of total influence of all CGH's parameters (parameters of the calculated IFD, parameters of e-beam litography, modes of electronic- and photoresists developing etc.) is extremely difficult and impossible in most cases.

The method of numerical modelling allows to bypass this difficulty and with use of a quite simple methods to receive exact quantitative results at any intermediate stage of holographic process.

A characteristic full enough for phase recording medium is a transmission function that can be presented as follows:

$$
t(\xi, \eta)=t_{0} \exp \left[i\left\{\psi_{n}(\xi, \eta)+\Delta \psi[\Delta E g(\xi, \mu)]\right\}\right] .
$$

For existing phase recording medium (electronic resist) it is possible to consider amplitude $t_{0}$ as a constant, phase variation $\Psi_{n}(\xi, \eta)$ caused by heterogeneity of a substrate causes only a slight alteration of the diffraction angle for the restoring beam, forming a low-frequency component of noise. A component $\Delta \Psi[\Delta E g(\zeta, \eta)]$ depends in the complex way on the changes of operating exposition $\Delta E g(\zeta, \eta)$ and can be expressed as:

$$
\begin{aligned}
& \Delta \Psi\left[\Delta E g(\zeta, \eta)=\frac{2 \pi}{\lambda}(n-1) L\left[g E n[r, s] \times h_{1}(\zeta, \eta) \times\right.\right. \\
& \left.\times h_{2}(\zeta, \eta)\right]=\frac{2 \pi}{\lambda}(n-1) L[\Delta L(\zeta, \eta)],
\end{aligned}
$$

where $g$ is a constant of this material describing a level of the dependence $\Delta L$ from $\Delta E ; n$ is a factor of medium refraction; $L$ is the operator considering nonlinearity of the relief-exposure characteristic; $\Delta L(\zeta, \eta)$-geometrical distribution relief modulation; $E n[r, s]$ - distribution of the imposed exposition (a calculated exposition of resolution elements of the hologram); $h_{1}(\zeta, \eta)-$ point spread function; $h_{2}(\zeta, \eta)$ - point spread function. When numerical modeling, we can assume $h(\zeta, \eta)=h_{1}(\zeta, \eta) \times$ $\times h_{2}(\zeta, \eta)$

Parameter of modulation $2 \pi / \lambda(n-1)$ that describes sensitivity of the recording medium during numerical modelling can be replaced with a parameter of the maximal phase shifting value $\Psi_{m}$, and the imposed exposition - distribution of phase shift $\Psi_{n}[r, s]$. In view of it, it is possible to write down the next expression:

$t(\xi, \eta)=\exp \left[i\left\{\psi\left[\psi_{m} \psi_{n}[r, s] \cdot h(\xi, \eta)\right]\right\}\right]$,

where $\Psi$ is the operator of transformation considering nonlinearity of the phase-exposing characteristic. Eq. (10) is the initial one to construct a digital model of the system.

Result of a synthesis operation is the matrix of numbers $T[r, s]$ that defines phase modulation for each element of the recorded hologram, the value of which is represented in (10) instead of $\Psi_{n}[r, s]$, thus $\Psi_{m} \Psi_{n}[r, s]$ varies from 0 up to $\Psi_{m}$. Taking into account that when modeling, only numerical analysis is used, so for performance of convolution (10), the continuous function $h(\zeta, \eta)$ should be replaced with its discrete analogue $h[p, g]$ with the step of digitization $\delta \zeta, \delta \eta$, defined according to the Nyiqist theorem. It is necessary to note that the function $h[p, g]$ and $T[r, s]$ have the different periods of digitization $\delta \zeta=\delta \eta<\Delta \zeta=\Delta \eta$. For the coordination of it, we set $\delta \zeta$ so $\Delta \zeta / \delta \zeta=P$, $\Delta \eta / \delta \eta=Q$, where $P$ and $Q$ are integers for the counts of the function $h[p, g]$ inside the cell $\Delta \zeta \times \Delta \eta$. As a result of performance of linear convolution the new sequence expressed below is formed:

$\Psi g[p, g]_{N P \times M Q}=T[r, s]_{N \times M} \times h[p, g]_{P \times Q}$.

After execution of the operation (10), the hologram is described by the following expression:

$t[p, g]=\exp \left[i\left\{\Psi\left[\Psi_{m} \Psi g[p, g]\right]\right\}\right]$.

The expression (12) is a digital model of a real digital hologram registered on the phase recording medium. When machine modelling of the recording medium and recording device, the following parameters were set:

$N=M=64 ; P=Q+8 ; \Psi_{m}=0-6.28 \mathrm{rad}$,

$h[p, g]$ was set by the table, its type was defined by the type of the recording medium;

[Y] was set by table, its type was defined by empiric data.

Analysis of the digital Fourier-hologram was carried out in the following way. The hologram designed according to (12) was lighted with normally falling flat 
wave with the amplitude $e=1$. Above the received distribution of field amplitudes, behind the hologram near to its plane, RFT was carried out using the FFT algorithms, thus there was a discrete distribution of the intensity in the plane of the restored image

$g[n, m]=\left|\mathrm{F}^{-1}\{\mathbf{t}[p, g]\}\right|^{2}$.

In view of periodicity of function $g[n, m]=$ $=g[n+Z \times P, m+Z \times Q]$, the calculation and the further analysis of the restored image was carried out only for one period $(\mathrm{Z}=0)$ - "zero" order of digitization with quantity of samples $N P \times M Q$.

For modelling the kinoform restoration processes, a program utilizing described algorithms was written. The block diagram of the program (Fig. 5) is shown below.

When modelling, boundary values for observation conditions (2) were explored. To define a necessity of condition of the phase matching, hologram restoration with different values of $c$ factor $(c<1, c=1, c>1)$ was simulated. Before restoration of the image, the array of the calculated phase values was multiplied by the factor $c$. At $c=1$, the phase matching condition is carried out, at $c<1$ or $c>1$, accordingly, it is not carried out. In Fig. 6, the results of modelling for different $c$ values are showed.

\subsection{Kinoform recording by electron-beam litography}

We used a method of electron-beam lithography for recording the kinoform on a phase recording medium (electron-sensitive PMMA resist).

When recording the kinoform, the use of the methods of lithograph digitization for the continuous function $\Delta \varphi(x, y)$ is required. So, it is replaced by a discrete function with the step $2 \pi / N$, where $N$ is a maximal possible number of levels for the phase relief in the given recording method. Thus, the diffraction efficiency is less than the theoretical one $100 \%$, but with growth of $N$ it increases - for example, at $N=2 ; 3$ or 10 the diffraction efficiency is equal accordingly $41 ; 81$ and $97 \%$. In our case, the quantity of levels is chosen to be equal to 16 (as a most simple way for realization).

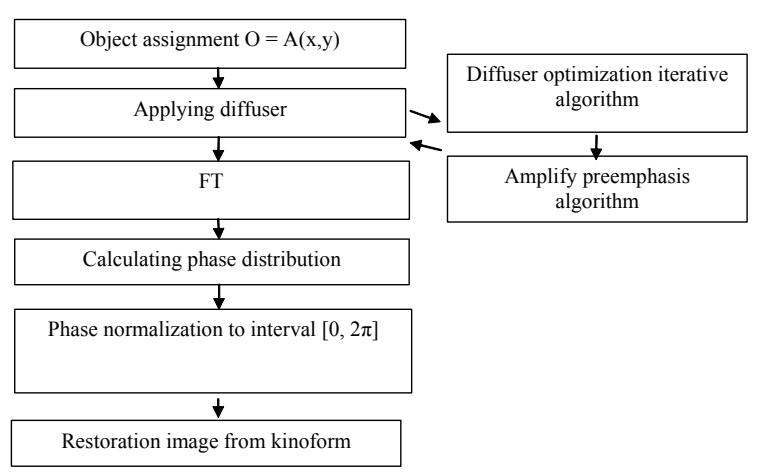

Fig. 5. Algorithm of synthesis and modelling the restoration of the kinoform hologram.
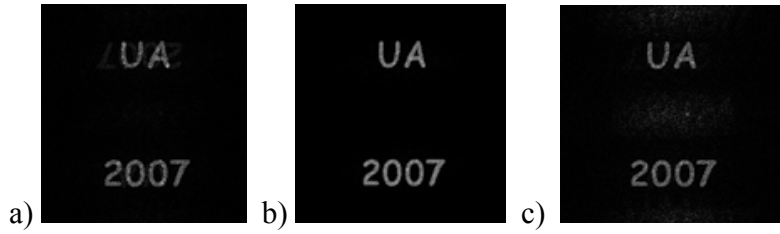

Fig. 6. Results of modelling for factor $c=0.7(a), c=1(b)$, $c=1.3(c)$.

Prepared in that way kinoform has the following reflection function:

$$
\begin{aligned}
& t(x, y) \sim \operatorname{rect}\left[\frac{x}{\Delta x}\right] \operatorname{rect}\left[\frac{y}{\Delta y}\right] \exp [ \pm i c \varphi(\bmod 2 \pi)] \times \\
& \times \sum_{n=-N}^{N} \sum_{m=-M}^{M} \delta(x-n \Delta x, y-n \Delta y) .
\end{aligned}
$$

The sign of exponent in the exponential factor is defined by that whether a negative or a positive image for kinoform is used. Accordingly, the image restored by kinoform will be real or virtual.

From consideration of the transmission function of the kinoform, it follows that for restoration of the initial wave front without distortions that the constant $c$ is required to be equaled to unity. It means that the light falling on the hologram sample with a phase $\tilde{\varphi}=0$, will delays equally for one wavelength in comparison with the light falling on the other sample with a phase $\tilde{\varphi}=2 \pi$. For example, in case of the reflective hologram for a sample with a phase $\tilde{\varphi}=2 \pi$ at the wavelength $\lambda=630 \mathrm{~nm}$, the depth of a maximal relief modulation will be equal to $\lambda / 2$, i.e. $315 \mathrm{~nm}$. If such phase matching has been achieved, all light falling on kinoform will participate in formation of the unique (real or imaginary) image of the object. Otherwise, the kinoform is similar to an axial hologram, where real and virtual images are partially superposed; some light diffracted into zero order will create a bright spot in the center of the image.

We preliminary recorded a series of tests to find pulse response characteristic of the recording system to choose optimum parameters for kinoform registration (Fig. 7).

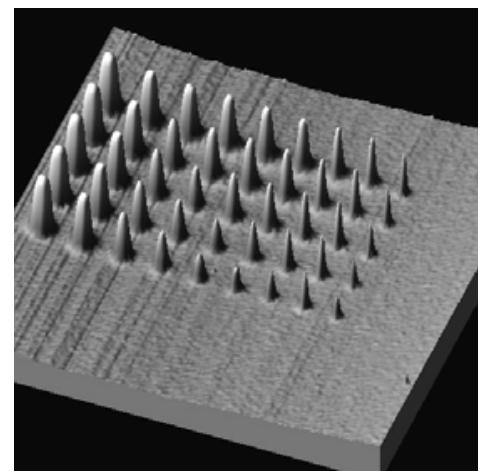

Fig. 7. Test structure, recorded for building the pulse-response curve of the recording system. 


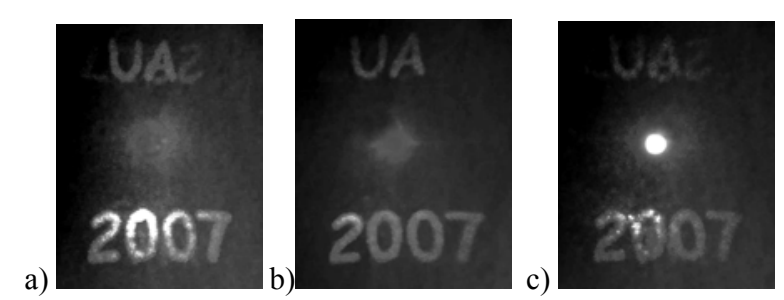

Fig. 8. Restored images $c=0.7$ (a), $c=1.3$ (b), $c=1$ (c).

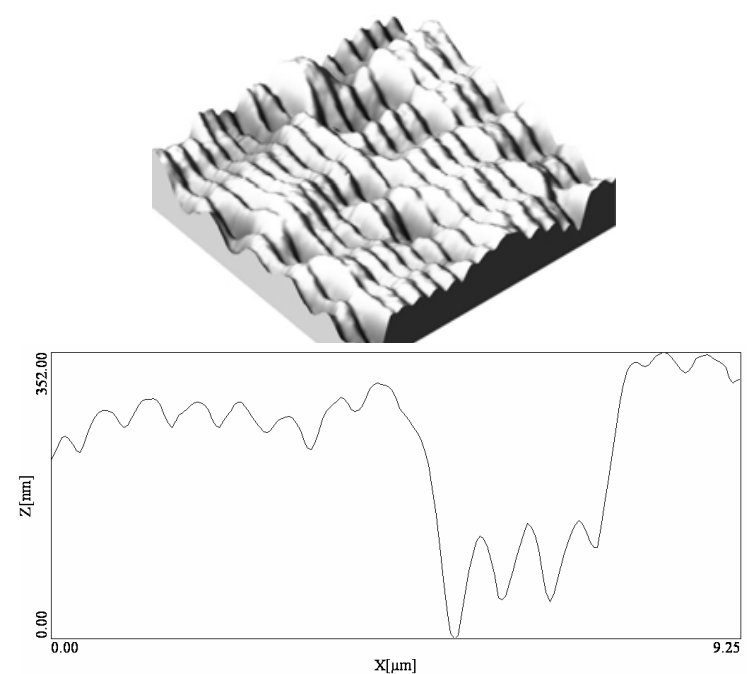

Fig. 9. Surface and profile of the kinoform (obtained with AFM ).

\subsection{Analysis of results}

As a result of using the described algorithms, test samples of the kinoform (with various values of parameter $c$ - less, more or equal to unity) have been recorded.

Photos of the restored images (Fig. 8) and the image of a phase relief (Fig. 9) made using an atomic force microscope are shown below.

\section{Conclusions}

In this paper, a problem of twin-image elimination in 2D digital holograms is considered. Two approaches to solve this problem are proposed: using quadrature holograms and kinoform methods. As shown above, use the kinoform for this purpose allows (without essential technology complexity of synthesis and record) to receive required result that is shown in photos of the images restored by recorded samples.

\section{References}

1. W.H. Lee, Sampled Fourier-transform hologram generated by computer // Appl. Opt. 9(3), p. 639643 (1970).

2. W.H. Lee, Computer-generated holograms: Techniques and application, Ed. E. Wolf. N.Y. // Progr. Opt. 16, p. 119-132 (1978).
3. L.P. Jaroslavski, N.S. Merzlyakov, Comparison of the methods of recording the computer-generated holograms // Abstr. Symp. “Optika-80”, 1980, p. 86.

4. D.C. Chu, J.F. Fienup, J.W. Goodman, Recent approaches to computer generated holograms // Opt. Eng. 13, (3), p. 189-195 (1974).

5. B.R. Brown, Computer synthesis of holograms and spatial filters // Application of holography, Ed. by Barrekette. N.Y.; L.1971, p. 215-227.

6. I.B. Lesem, P.M. Hirch, J.A. Jordan. The kinoform a new wave-front reconstruction device // IBM Journal of Research and Develop. 13, 2 (1969).

7. L.M. Soroko, Gilbert-optics. M., 1981, p. 21-25.

8. D. Gabor, W. Goss, Interference microscope with total wave-front reconstruction // J. Opt. Soc. Amer. 56, No 7, p. 849 (1966).

9. Baltiyskiy et al., Contemporary methods of digital holography. In: Problems of coherent and nonlinear optics, S.-Pt., 2004, p. 91-117.

10. E. Cuche, P. Marquet, Ch. Depeursinge, Spatial filtering for zero-order and twin-image elimination in digital off-axis holography // Applied Optics 39, No. 23, 2000.

11. A.I. Fishman, The phase optical elements kinoforms // Soros Review Journal (Sorosovskij obrazovatel'nyj zhurnal) No. 12, p. 76-83, 1999.

12. T.I. Kuznecova, About the phase problem in optics // Uspekhi fiz. nauk 154(4), p. 677-690 (1988).

13. B. Chhetri, S. Serikawa, S. Yang, et al., Binary phase difference diffuser: Design and application in digital holography // Opt. Eng. 41(2), p. 319-327, 2002.

14. V.I. Girnyk, V. Kurashov, A. Makarovsky, Synthesis of a kinoform with improved intensity transmission in restored image. Proc. 3-rd Union School on Holography - Riga, 1980, part II, p. 274.

15. V.I. Girnyk, V. Kurashov, A. Makarovsky, Amplitude preamphasis algorithm. Proc. 3-rd Union School on Holography. - Riga, 1985, p. 315-316.

16. V. Girnyk, S. Kostyukevych, A. Kononov, I. Borisov, Multilevel computer-generated holograms for reconstructing 3D images in combined opticaldigital security devices // SPIE Proc. 4677, p. 255 266, 2002.

17. V. Girnyk, S. Kostyukevych, E. Braginets, A. Soroka, 3D $\mathrm{CGH}$ registration on organic and nonorganic resists: comparative analysis // SPIE Proc. 6136, p. 6136OP, 2006.

18. E.V. Braginets, V.I. Girnyk, S.A. Kostyukevych, Computer-generated holograms of 3D images in optical security devices // SPIE Proc. 5742, p. 33 40, 2005.

19. V.I. Girnyk, S.O. Kostyukevich, P.Ye. Shepeliavyi, Multilevel computer-generated holograms for reconstructing 3-D images in combined opticaldigital security devices // Semiconductor Physics, Quantum Electronics \& Optoelectronics 5(1), p. 106-114 (2002). 